\title{
B2 ordering in body-centered-cubic AINbTiV refractory high-entropy alloys
}

\author{
Fritz Körmann $\odot,{ }^{1,2, *}$ Tatiana Kostiuchenko $\odot,{ }^{3}$ Alexander Shapeev $\odot,^{3}$ and Jörg Neugebauer $\oplus^{1}$ \\ ${ }^{1}$ Computational Materials Design, Max-Planck-Institut für Eisenforschung GmbH, D-40237 Düsseldorf, Germany \\ ${ }^{2}$ Materials Science and Engineering, Delft University of Technology, 2628 CD, Delft, The Netherlands \\ ${ }^{3}$ Skolkovo Institute of Science and Technology, Skolkovo Innovation Center, Nobel St. 3, Moscow 143026, Russia
}

(Received 4 December 2020; accepted 11 May 2021; published 24 May 2021)

\begin{abstract}
The phase stability of a bcc AlNbTiV high-entropy alloy at elevated temperatures is studied using a combination of machine-learning interatomic potentials, first-principles calculations, and Monte Carlo simulations. The simulations reveal a B2 ordering below about $1700 \mathrm{~K}$, mainly caused by a strong site preference of $\mathrm{Al}$ and Ti. A much weaker site preference for $\mathrm{V}$ and $\mathrm{Nb}$ is observed, strongly affecting the alloys total configurational entropy. The underlying mechanisms of the B2 phase stability as opposed to the random solid solution are discussed in terms of a high persisting configurational entropy of the B2 phase due to strong sublattice site disorder.
\end{abstract}

DOI: 10.1103/PhysRevMaterials.5.053803

\section{INTRODUCTION}

After refractory high-entropy alloys were proposed a decade ago by Senkov et al. [1-3], there has been recent interest in exploring the possibility of so-called refractory high-entropy superalloys (RSAs) [4]. These are multicomponent alloys, often referred to as compositionally complex or high-entropy alloys, containing refractory elements and featuring dual-phase microstructures with disordered bcc A2 and ordered B2 phases. This combination could result in improved mechanical properties as provided for their fcc Ni-superalloy counterpart. Given the relevance of conventional superalloys for manifold technological applications, the huge compositional phase space provided by the RSA design strategy promises tremendous opportunities for exploring and designing alternative alloys.

Often $\mathrm{Al}$ is added to form a B2 phase in bcc refractory multicomponent alloys. This has beneficial side effects, such as reducing the alloy density and improving oxidation resistance. Various potential RSAs have been found in the last decade featuring dual A2+B2 phases [5] (see also Ref. [4] for a recent overview). Among them are various Al-containing refractory multicomponent alloys. The B2 phase formation in Al-containing bec multicomponent refractory alloys is, however, still intriguing, since in many Al binaries with refractory elements, B2 phases are typically not observed.

For this reason, conventional thermodynamic databases are often not suitable to predict B2 phase formation in Alcontaining bcc refractory multicomponent alloys due to scarce

\footnotetext{
*koermann@mpie.de
}

Published by the American Physical Society under the terms of the Creative Commons Attribution 4.0 International license. Further distribution of this work must maintain attribution to the author(s) and the published article's title, journal citation, and DOI. Open access publication funded by the Max Planck Society.
B2 formability of the sub-binaries in these alloys. Experimental identification of B2 is also often challenging. In bcc MoCrTiAl, it has been shown that interpreting x-ray diffraction patterns is not sufficient to identify a potential B2-type crystal structure [6]. Another example is bcc AlNbTiV, which first has been characterized as a disordered A2 phase [7,8]. Due to the mentioned challenges, a thermodynamic analysis utilizing the Calphad approach [9] corroborated this initial assessment [8]. However, later experimental analyses [10,11] have shown that bcc AlNbTiV features a single B2 phase over a wide temperature range. Based on earlier studies on $\mathrm{Ti}$ alloys, it was suggested [11] that $\mathrm{Al}$ and $\mathrm{Nb}$ as well as $\mathrm{Ti}$ and $\mathrm{V}$ occupy the two sublattice sites, i.e., B2(Al,Nb) (Ti,V). However, the underlying mechanism stabilizing the B2 phase as well as the detailed site occupancies still remain unclear. One hypothesis is that a considerable sublattice disorder may still be present, thus stabilizing the B2 phase in these multicomponent alloys [4]. A key to explore and design such RSAs featuring B2 phases is therefore to first reveal the underlying mechanisms responsible for the B2 phase stability and explore the detailed site concentrations.

In this work we systematically investigate the B2 ordering in the prototypical bcc AlNbTiV alloy by performing an extensive first-principles-based investigation. Specifically, we employ density-functional theory (DFT) calculations in combination with a machine-learning potential [12-14] and Monte Carlo simulation to investigate the short-range order and phase stability. The presence of a B2 phase, in particular, the site occupancies and underlying reasons for the thermodynamic stability, are discussed.

\section{METHOD AND COMPUTATIONAL DETAILS}

Simulations are performed employing the low-rank interatomic potential (LRP) [12] method. This is an on-lattice machine-learning potential, particularly suited to fit multicomponent alloys [12] and which has been also recently utilized to study chemical short-range order in bcc NbMoTaW 
[13] and fcc VCoNi [14] alloys. The LRPs in this work are used in canonical Monte Carlo (MC) simulations to explore the phase stability.

The LRPs are fitted to density-functional theory calculations which have been carried out employing the VASP code [15-18]. The projector augmented wave (PAW) method [19] within the Perdew-Burke-Ernzerhof generalized gradient approximation (PBE-GGA) [20] has been used.

To include the impact of volume expansion, the temperature expansion has been computed based on a DebyeGrüneisen model [21]. For this the total energy of the random alloy, $E(V)$, its bulk modulus and the bulk modulus derivative are computed based on a $4 \times 4 \times 4$ (128-atom) special quasi-random structure (SQS) [22]. The derived zero-K and predicted room-temperature lattice constants are 3.187 and $3.200 \AA$, being close to the reported experimental value $[7,11]$. To include the impact of thermal expansion, the calculations for fitting the LRP are conducted at a lattice parameter of $3.23 \AA$, corresponding to the computed value at around $1000 \mathrm{~K}$. Note that the computation of more accurate vibrational free energies such as, e.g., performed in Refs. [23,24], may quantitatively alter the computed phase transition temperature, but is beyond the scope of the present work.

A plane-wave cutoff energy of $450 \mathrm{eV}$ has been chosen. The $k$-point meshes generated by the Monkhorst-Pack [25] scheme were set to $2 \times 4 \times 4,5 \times 5 \times 5$, and $5 \times 5 \times 5$ for supercell sizes of $8 \times 4 \times 4,4 \times 4 \times 4$, and $5 \times 5 \times 5$ (containing 256,128 , and 250 atoms, respectively), corresponding to $k p$ densities of 8192,8192 , and $16,000 \mathrm{kp}$ atom. The convergences for the electronic minimization and relaxation of internal atomic positions were set to thresholds below $5 \times 10^{-3}$ and $5 \times 10^{-4} \mathrm{meV} /$ atom while keeping the cubic shape fixed. A smearing parameter of $0.12 \mathrm{eV}$ was chosen with the Fermi smearing method to include the impact of electronic excitations.

The initial data set for the machine-learning potentials included 200 random configurations for the 250 -atom $(5 \times$ $5 \times 5$ ) bcc supercell. The training set consists of $90 \%$ of this data, and $10 \%$ was utilized for the validation set. The fitting and validation errors of these initial LRPs are around $2 \mathrm{meV} /$ atom. Different ranks for the LRP were evaluated, and a rank of 4 was chosen for the initial retraining phase. No significant improvement on the fitting and validation error was observed for choosing a higher rank at this stage.

A set of ten independent LRPs were fitted and utilized in subsequent Monte Carlo simulations for $5 \times 5 \times 5$ supercells for the retraining procedure. For temperature ranges where significant variations among the different potentials were observed, new training configurations were selected from MC snapshots. For the present work we retrained the LRPs at 13 temperatures, selected in regimes where the strongest fluctuations among the initial potentials and $\mathrm{MC}$ results were observed. For each temperature a set of ten configurations was added to the training set. After performing two rounds of retraining we added one further round of retraining on $8 \times 4 \times 4$ supercells. The final training and validation set contained 547 and 60 DFT calculations, respectively. As the training data had been significantly increased during the retraining process, the rank of the potential was optimized and we found that a rank of 6 provides the best balance of training and validation

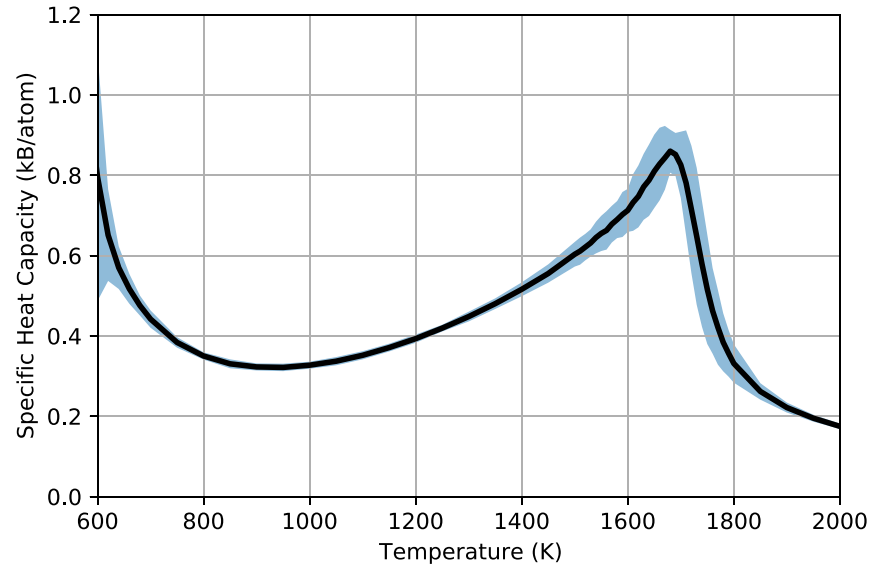

FIG. 1. Specific heat capacity, $C_{V}(T)$, vs temperature as derived from the Monte Carlo simulations for bcc AlNbTiV from a $14 \times 14 \times 14$ simulation box containing 5488 atoms. The peak at around $1700 \mathrm{~K}$ corresponds to an A2-B2 phase transition. The increase at temperatures below $800 \mathrm{~K}$ indicates the inset of another phase transition. The shaded area indicates twice the standard deviation provided by the ten independent LRPs.

error of about 1.7 and $1.9 \mathrm{meV} /$ atom, respectively. All ten independently fitted potentials, used for the actual analysis in the remainder of this work, revealed similar performance (see also Fig. 6 in the Appendix).

The majority MC simulations were performed for supercells containing 5488 atoms $(14 \times 14 \times 14$ supercells based on the two-atom primitive bcc cell) with periodic boundary conditions. The burn-in approach [26] was used, neglecting the first half of MC steps for each temperature value and thus improving the robustness of the algorithm. For temperatures higher than $2100 \mathrm{~K}, 2 \times 10^{6} \mathrm{MC}$ steps and for all other temperatures $2 \times 10^{7} \mathrm{MC}$ steps were chosen, where each MC step represents one swap attempt for every atom (see Appendix). For the MC simulations utilized to generate the set for retraining, 250 and 256 atoms $(5 \times 5 \times 5$ and $8 \times 4 \times 4$ supercells $)$ were chosen.

\section{RESULTS AND DISCUSSION}

We first discuss the specific heat capacity, $C_{V}(\mathrm{~T})$, as obtained from the MC simulations. The results are shown in Fig. 1. The accuracy of the retrained LRPs is exemplified by the shaded area, which represents twice the standard deviation of the $\mathrm{MC}$ results from the ten independently fitted potentials. The observed peak at around $1700 \mathrm{~K}$ indicates a phase transition which is identified as an A2-B2 transition further below. This B2 phase is stable down to about $550 \mathrm{~K}$. The increase in $C_{V}(\mathrm{~T})$ for temperatures below $800 \mathrm{~K}$ indicates another phase transition at around $500-550 \mathrm{~K}$. A preliminary inspection of a MC snapshot at $480 \mathrm{~K}$ (not shown) indicates a decomposition, into (at least) two phases, one enriched in V. We do not discuss here the phase stability at lower temperatures, as this would be beyond the scope of the present work. The observed range of B2 stability is also consistent with annealing experiments at $1273 \mathrm{~K}$ [10] and $1473 \mathrm{~K}$ [11], where a B2 phase has been reported. 


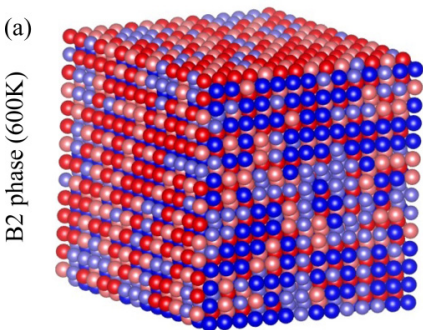

(b)
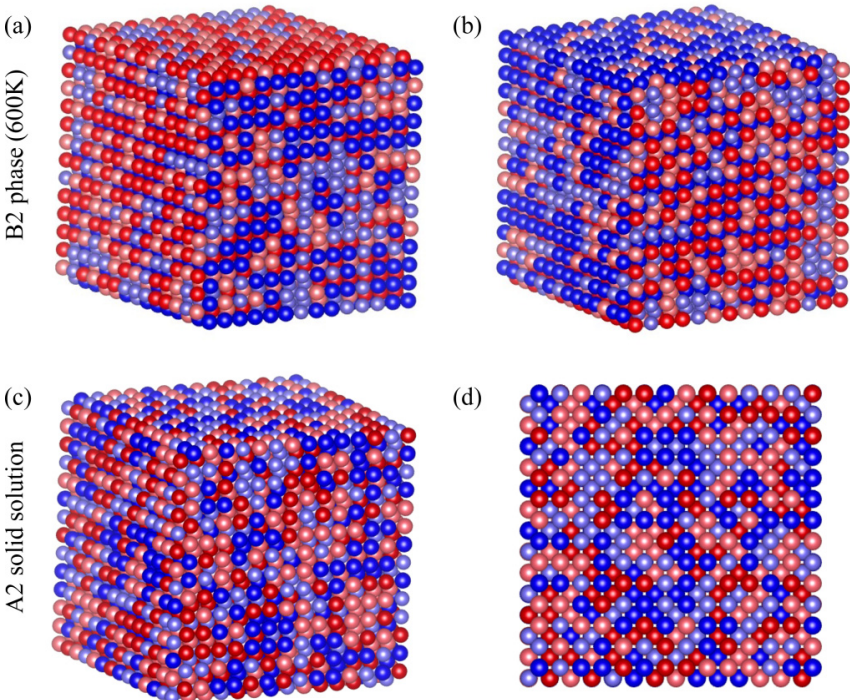

(d)

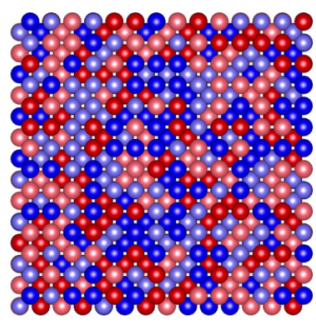

FIG. 2. (a), (b) Snapshot of the employed supercells from the Monte Carlo simulations for the B2-ordered alloy (at $600 \mathrm{~K}$ ) for two side views. (c), (d) Top and side view for a fully random solid solution. The B2 ordering in (a, b) is mainly driven by $\mathrm{Al}$ (red) and $\mathrm{Ti}$ (blue), whereas $\mathrm{Nb}$ and $\mathrm{V}$ (light red and light blue) reveal a weaker sublattice site preference. Figures created by OVITO [27].

To elucidate the ordering, we present in Figs. 2(a) and 2(b) a snapshot from a MC simulation at $600 \mathrm{~K}$ featuring a B2 ordering and as comparison in (c, d), an example for an ideal random solid solution. In contrast to the completely random state at high temperatures, a B2 ordering is clearly visible in Fig. 2(a), driven mainly by $\mathrm{Al}$ (red) and $\mathrm{Ti}$ (blue) atoms.

To explore the ordering tendencies more quantitatively, we discuss next the first- and second-shell pair-correlation parameters, often referred to as the Warren-Cowley shortrange order (SRO) parameters [28] as derived from the MC simulations,

$$
\alpha_{m}^{i j}=1-\frac{p_{m}^{i j}}{c_{i} c_{j}}
$$

where $\alpha_{m}^{i j}$ is the Warren-Cowley SRO parameter for atom $i$ and $j$ in the $m$ th shell, $p_{m}^{i j}$ is a probability of finding atom $j$ at the $m$ th shell of atom $i$, and $c_{i}, c_{j}$ are the total concentrations of the $i$ and $j$ elements in the alloy. Vanishing $\alpha$ values describe the random alloy, a positive value indicates clustering or segregation, and negative values indicate attraction of $i$ and $j$ atoms.

The SRO parameters, directly related to the paircorrelation parameters according to Eq. (1) above, are shown for the first and second shell in Figs. 3(a) and 3(b), respectively. Most striking is the strong positive (attractive) Al-Ti SRO parameter for the first shell and negative (repulsive) one in the second shell (both in blue). This clearly underlines that the main contribution to SRO above the ordering temperature is driven by favorable Al-Ti pairs in the first and repulsive ones in the second shell, consistent with a B2 ordering. Below $1700 \mathrm{~K}$, an increasing probability for $\mathrm{Al}-\mathrm{V}$ and $\mathrm{Ti}-\mathrm{Nb}$ in the first shell and $\mathrm{Al}-\mathrm{Nb}$ as well as Ti-V favorable pairs for
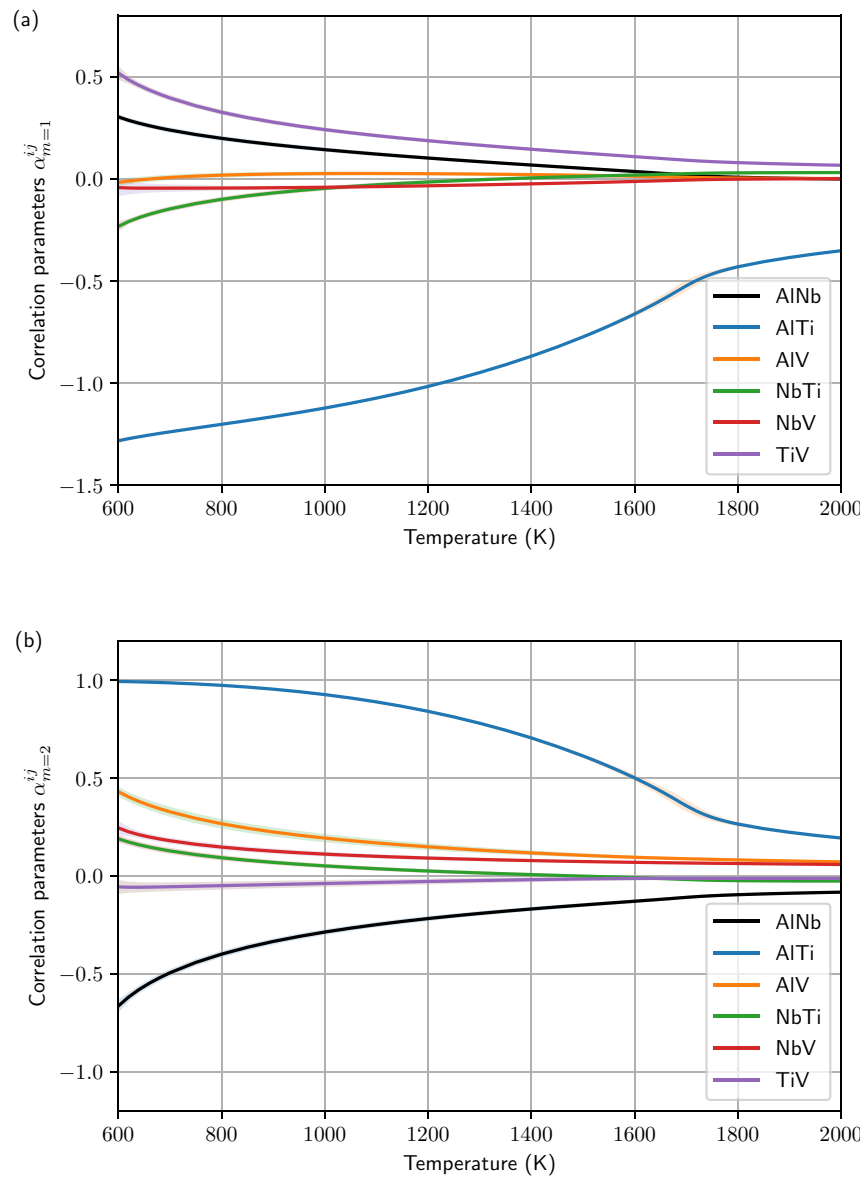

FIG. 3. Warren-Cowley parameters $\alpha_{m}^{i j}$ (related to the paircorrelation parameters) for the (a) first $(m=1)$ and (b) second shell $(m=2)$ from Monte Carlo simulations for bcc AlNbTiV. A strong $\mathrm{Al}-\mathrm{Ti}$ attraction in the first shell and repulsion in the second shell is present, also in the solid solution at high temperatures. Shaded areas indicate twice the standard deviation provided by the ten independent LRPs.

the second shell are found. This indicates the tendency for enrichment of $\mathrm{Al}$ and $\mathrm{Ti}$ sites with $\mathrm{Nb}$ and $\mathrm{V}$, respectively.

In order to explore the underlying site occupations in more detail, we analyzed the local chemical concentration of the elements on the two sublattice sites of the B2 phase [see sketch in Fig. 4(a)]. The sublattice occupations can be interpreted as an order parameter for the B2 phase (see, e.g., Ref. [29] for a recent application to a bcc high-entropy alloy). The results for one of the sublattice sites are shown in Fig. 4(b). The site occupancies clearly reflect a B2 ordering setting at around $1700 \mathrm{~K}$. In particular, $\mathrm{Al}$ and $\mathrm{Ti}$ are driving the site occupations. At about $1000 \mathrm{~K}, \mathrm{Al}$ is almost completely occupying one of the sublattices, whereas $\mathrm{Ti}$ is completely depleted, occupying mainly the other sublattice site (not shown here). As found for the SRO parameters, there is also the tendency for $\mathrm{Nb}$ to mix on the $\mathrm{Al}$ and for $\mathrm{V}$ to mix on the Ti sites. The enrichment of the $\mathrm{Al}$ sites with $\mathrm{Nb}$ and $\mathrm{Ti}$ with $\mathrm{V}$ is, however, over a wide temperature range very weak. For example, at $1000 \mathrm{~K}$, where $\mathrm{Al}$ already occupies one sublattice site with over $49 \%$, the $\mathrm{Nb}$ and $\mathrm{V}$ concentration on this Al-rich site is about $30 \%$ and $20 \%$. This implies a strong sublattice disorder 

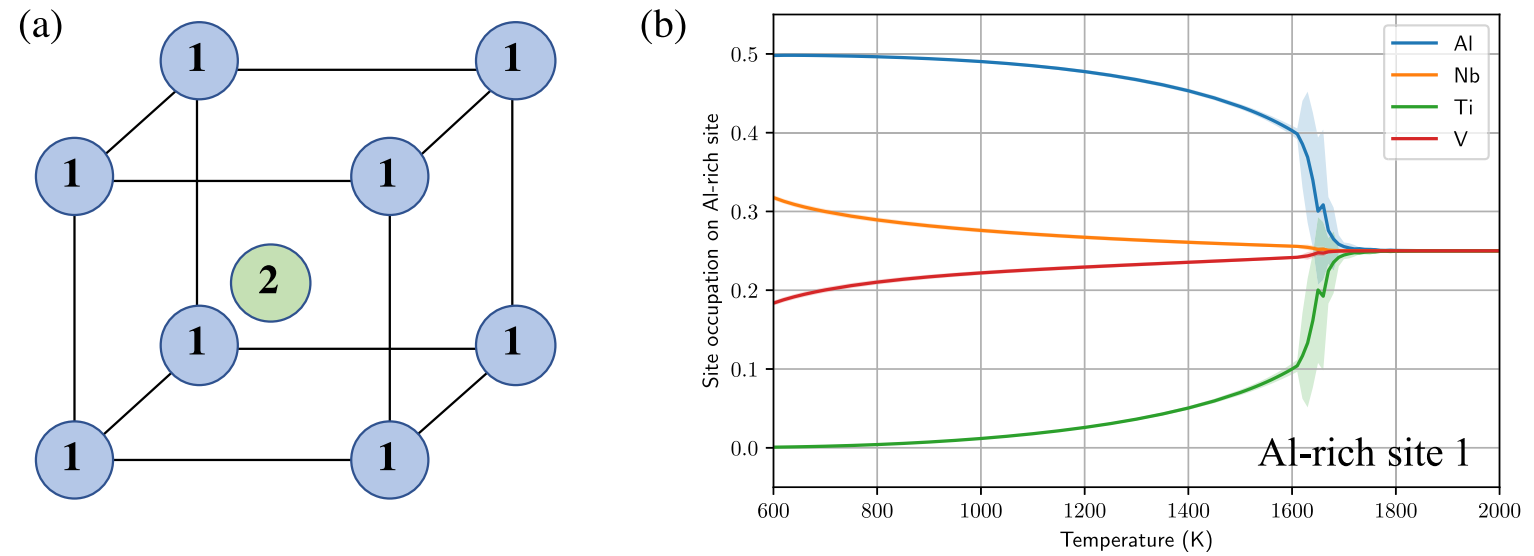

FIG. 4. (a) Sketch for a B2 ordering with distinct sites 1 and 2. (b) Sublattice occupation of the Al-rich site in the B2 phase derived from Monte Carlo simulations for AlNbTiV. The $\mathrm{B} 2$ ordering is mainly driven by $\mathrm{Al}$ and $\mathrm{Ti}$, with a tendency for $\mathrm{Nb}$ to mix on the $\mathrm{Al}$ sublattice and for $\mathrm{V}$ to mix on the Ti sublattice. Shaded areas indicate twice the standard deviation provided by the ten independent LRPs.

and hence a considerable configurational entropy persisting in the $\mathrm{B} 2$ phase.

To elucidate the impact of the persisting sublattice disorder in the B2 phase on the phase stability we computed the configurational entropy. This has been done by integrating $C_{V}(T)$ from high temperatures and subtracting it from the known limit of the random solid solution. The results are shown in Fig. 5. Consistent with the sublattice occupation, we find a relatively large configurational entropy of the B2 phase, mainly driven by the intermixed $\mathrm{Nb}$ and $\mathrm{V}$ atoms on the sublattices. To put the results into perspective, we also show the configurational entropy of the ideal random solid solution, of an ideal B2(AlNbV)(TiNbV) phase, and of an ideal $\mathrm{B} 2(\mathrm{AlNb})(\mathrm{TiV})$ phase. The latter two scenarios provide two idealized $\mathrm{B} 2$ scenarios, with $\mathrm{Nb}$ and $\mathrm{V}$ having no site preference, and with $\mathrm{Nb}$ and $\mathrm{V}$ completely occupying $\mathrm{Al}$ and $\mathrm{Ti}$ sites. Indeed, the computed entropy at $1000 \mathrm{~K}$ is close to

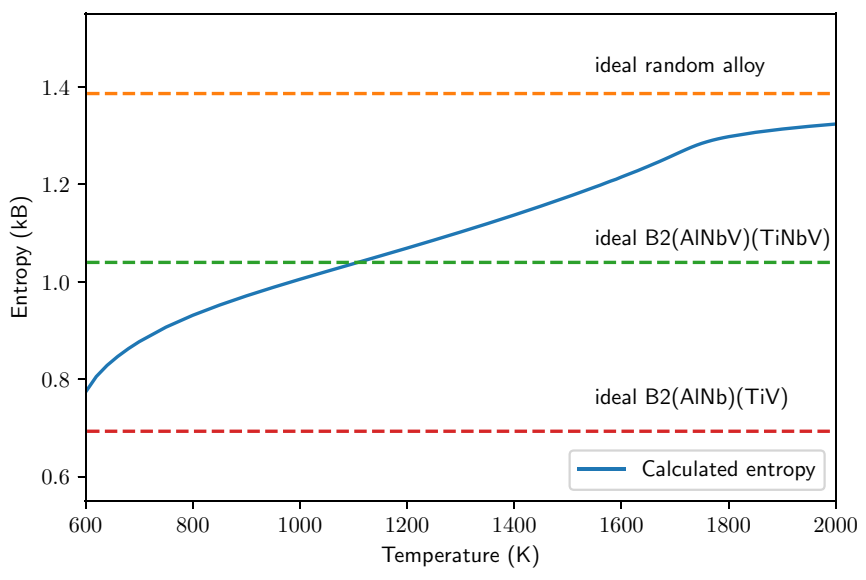

FIG. 5. Configurational entropy vs temperature obtained by integrating the specific heat capacity shown in Fig. 1. As a comparison the configurational entropy of the ideal random solid solution, a hypothetical B2(AlNbV)(TiNbV) and a B2(AlNb)(TiV), are shown. The directly computed entropy is over a large temperature range much larger as compared to an idealized B2(AlNb)(TiV) scenario. the scenario of a B2(AlNbV)(TiNbV) phase with almost no site preference of $\mathrm{Nb}$ and $\mathrm{V}$. It is also about $50 \%$ larger as compared to the entropy of an ideal $\mathrm{B} 2(\mathrm{AlNb})(\mathrm{TiV})$ phase, where $\mathrm{Nb}$ and $\mathrm{V}$ are occupying only $\mathrm{Al}$ and $\mathrm{Ti}$ sites, respectively. This can have important consequences in modeling the configurational entropy and interpreting experiments. For example, in [11] the local ordering in bcc AlNbTiV has been analyzed by assuming a B2(AlNb)(TiV) sublattice ordering. For bec AlTiVCr [30], a B2(AlCr)(TiV) phase has been ad hoc assumed in atomistic simulations due to the lack of further SRO information. However, as shown in the present case, such assumptions could largely overestimate the configurational entropy contribution and thus bias the predicted phase transition temperature. In fact, various bcc multicomponent alloys including $\mathrm{Al}$ and $\mathrm{Ti}$ have been proposed in the last years, among them, e.g., bcc AlNbTiV, AlHfNbTi, AlHfTaTi, and AlMoNbTi, all of which show a single B2 phase $[6,11,31]$. Given the similarity in these alloys, it is possible that similar strong sublattice disorder may also persist in the observed B2 phase in these alloys.

\section{CONCLUSION}

Chemically complex bcc alloys, containing A2-B2 dualphase structure, may open the way to design refractory high-entropy superalloys. The B2 phase stability plays therefore a crucial role. The underlying reason for the B2 phase stability in these Al-containing refractory alloys has been unclear, and detailed sublattice ordering information were, however, lacking so far. We employed a combination of firstprinciples informed, machine-trained interatomic potentials and Monte Carlo simulations to investigate the ordering and phase stability of the prototypical bcc AlNbTiV alloy. A single $\mathrm{B} 2$ phase is revealed below $1700 \mathrm{~K}$, mainly driven by $\mathrm{Al}$ and $\mathrm{Ti}$, with a weak tendency for $\mathrm{Nb}$ and $\mathrm{V}$ enrichment on the $\mathrm{Al}$ and $\mathrm{Ti}$ sites. As a result, the derived configurational entropy is about 50\% larger than one may intuit from an ad hoc assumed B2(AlNb)(TiV) scenario. Given the many proposed bcc multicomponent alloys containing $\mathrm{Al}$ and $\mathrm{Ti}$, the obtained insights of the present study may be also valid 


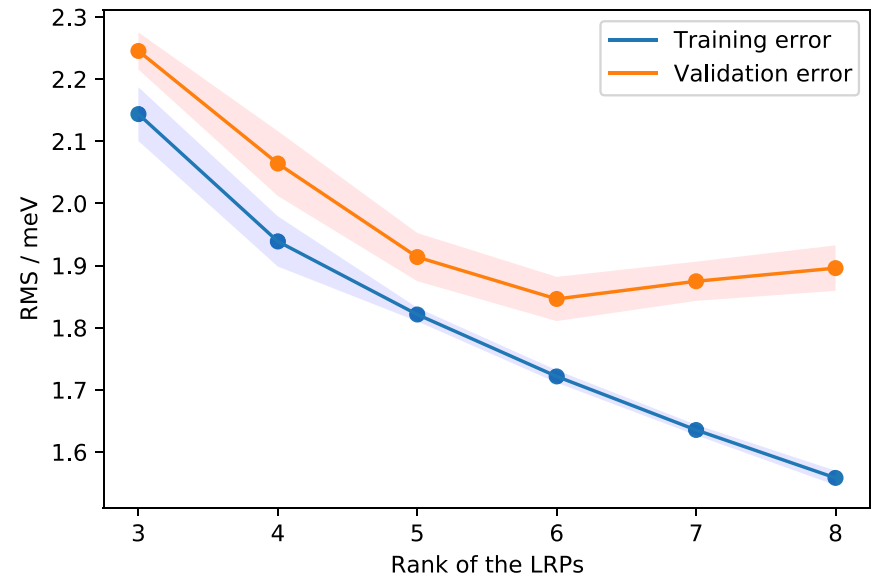

FIG. 6. The mean training and validation error vs the rank. The mean errors were averaged over ten independently fitted LRPs, and the shaded area indicates the standard deviation among these potentials. A rank of 6 was eventually chosen for the final calculations.

for various other alloys too, highlighting the significant role of detailed SRO and phase stability explorations for modeling and designing Al-containing bec concentrated complex alloys.

The datasets generated during the current study are available from the corresponding author on reasonable request.

\section{ACKNOWLEDGMENTS}

We gratefully acknowledge stimulating discussions with Konstantin Gubaev and Marcel Sluiter. Funding from the German Research Foundation DFG within the priority program SPP 2006, a collaborative German Research Foundation DFG and Russian Foundation for Basic Research RFBR grant (No. DFG KO 5080/3-1 and RFBR Grant No. 20-53-12012), and the NWO/STW (VIDI Grant No. 15707) are gratefully acknowledged.

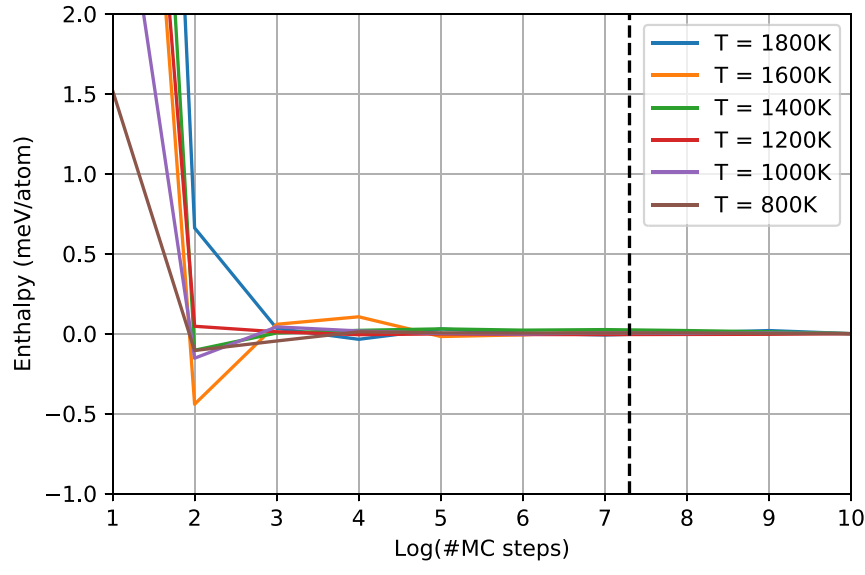

FIG. 7. The convergence of the enthalpy at different temperatures obtained for a given LRP is shown vs the number of MC steps (in logarithmic scale). The chosen number of MC steps for further analysis is indicated as a dashed line.

\section{APPENDIX}

Here we provide several technical details and convergence tests with respect to the chosen rank of the potential and MC parameters. Ten potentials were independently fitted to the training and validation set containing 547 and 60 configurations, respectively. In Fig. 6 the achieved training and validation errors are shown versus the chosen rank of the potential. From this analysis a rank of 6 has been determined, providing an optimal balance of training and validation error.

The number of MC steps has been evaluated as part of the convergence tests. In Fig. 7 the convergence of the enthalpy as derived from the MC simulations versus the number of utilized MC steps is shown for various temperatures. The dashed line corresponds to the eventually chosen number of $\mathrm{MC}$ steps for the majority of the MC simulations.
[1] O. N. Senkov, G. Wilks, J. Scott, and D. B. Miracle, Intermetallics 19, 698 (2011).

[2] O. Senkov, J. Scott, S. Senkova, D. Miracle, and C. Woodward, J. Alloys Compd. 509, 6043 (2011).

[3] O. Senkov, G. Wilks, D. Miracle, C. Chuang, and P. Liaw, Intermetallics 18, 1758 (2010).

[4] D. B. Miracle, M.-H. Tsai, O. N. Senkov, V. Soni, and R. Banerjee, Scr. Mater. 187, 445 (2020).

[5] V. Soni, O. N. Senkov, B. Gwalani, D. B. Miracle, and R. Banerjee, Sci. Rep. 8, 8816 (2018).

[6] H. Chen, A. Kauffmann, S. Seils, T. Boll, C. Liebscher, I. Harding, K. Kumar, D. Szabó, S. Schlabach, S. KauffmannWeiss et al., Acta Mater. 176, 123 (2019).

[7] N. Stepanov, D. Shaysultanov, G. Salishchev, and M. Tikhonovsky, Mater. Lett. 142, 153 (2015).

[8] N. Stepanov, N. Y. Yurchenko, D. Skibin, M. Tikhonovsky, and G. Salishchev, J. Alloys Compd. 652, 266 (2015).

[9] The thermodynamic analysis in Ref. [8] has been performed using the TTTI3 (titanium alloys) database. Also a $\mathrm{Ti}_{3} \mathrm{Al}$ phase below $1043 \mathrm{~K}$ has been predicted.

[10] N. Y. Yurchenko, N. Stepanov, A. Gridneva, M. Mishunin, G. Salishchev, and S. Zherebtsov, J. Alloys Compd. 757, 403 (2018).

[11] N. Y. Yurchenko, N. Stepanov, S. Zherebtsov, M. Tikhonovsky, and G. Salishchev, Mater. Sci. Eng. A 704, 82 (2017).

[12] A. Shapeev, Comput. Mater. Sci. 139, 26 (2017).

[13] T. Kostiuchenko, F. Körmann, J. Neugebauer, and A. Shapeev, npj Comput. Mater. 5, 55 (2019).

[14] T. Kostiuchenko, A. V. Ruban, J. Neugebauer, A. Shapeev, and F. Körmann, Phys. Rev. Mater. 4, 113802 (2020).

[15] G. Kresse and J. Hafner, Phys. Rev. B 47, 558 (1993).

[16] G. Kresse and J. Hafner, Phys. Rev. B 49, 14251 (1994).

[17] G. Kresse and J. Furthmüller, Comput. Mater. Sci. 6, 15 (1996).

[18] G. Kresse and J. Furthmüller, Phys. Rev. B 54, 11169 (1996).

[19] P. E. Blöchl, Phys. Rev. B 50, 17953 (1994). 
[20] J. P. Perdew, K. Burke, and M. Ernzerhof, Phys. Rev. Lett. 77, 3865 (1996).

[21] V. L. Moruzzi, J. F. Janak, and K. Schwarz, Phys. Rev. B 37, 790 (1988)

[22] A. Zunger, S.-H. Wei, L. G. Ferreira, and J. E. Bernard, Phys. Rev. Lett. 65, 353 (1990).

[23] F. Körmann, Y. Ikeda, B. Grabowski, and M. H. Sluiter, npj Comput. Mater. 3, 36 (2017).

[24] B. Grabowski, Y. Ikeda, P. Srinivasan, F. Körmann, C. Freysoldt, A. I. Duff, A. Shapeev, and J. Neugebauer, npj Comput. Mater. 5, 80 (2019).

[25] H. J. Monkhorst and J. D. Pack, Phys. Rev. B 13, 5188 (1976).
[26] M. K. Cowles and B. P. Carlin, J. Am. Stat. Assoc. 91, 883 (1996).

[27] K. Momma and F. Izumi, J. Appl. Crystallogr. 44, 1272 (2011).

[28] J. Cowley, Phys. Rev. 120, 1648 (1960).

[29] L. J. Santodonato, P. K. Liaw, R. R. Unocic, H. Bei, and J. R. Morris, Nat. Commun. 9, 4520 (2018).

[30] Y. Qiu, Y. Hu, A. Taylor, M. Styles, R. Marceau, A. Ceguerra, M. Gibson, Z. Liu, H. Fraser, and N. Birbilis, Acta Mater. 123, 115 (2017).

[31] F. G. Coury, T. Butler, K. Chaput, A. Saville, J. Copley, J. Foltz, P. Mason, K. Clarke, M. Kaufman, and A. Clarke, Mater. Des. 155, 244 (2018). 